\title{
Exploring gender differences in Malaysian urban adolescent Internet usage
}

\begin{abstract}
This study explored gender differences in urban adolescent Internet access, usage and motives. Data were collected from 914 urban school students in Malaysia. Factor analysis revealed that eroticism, entertainment, social-interaction, shopping and information/ surveillance are the key drivers for adolescence Internet usage. No differences between boys and girls were detected in Internet accessibility and home computer ownership. Boys and girls differed in their intensity of usage, place of access and their motivations to use the Internet. Girls were more motivated by social-interaction, shopping and surveillance/ information, while boys were more motivated by eroticism and had a higher tendency to be addicted to the Internet. However, boys and girls did not exhibit any significant differences in online entertainment motivation.
\end{abstract}

Keyword: Uses \& gratifications; Internet; Gender 